\title{
Otimização para controle de alagamentos urbanos: aplicação na bacia hidrográfica Vila Santa Isabel/Viamão/RS
}

O crescimento rápido e descontrolado da urbanização, principalmente nos países em desenvolvimento, vem provocando impactos significativos tanto no meio ambiente quanto para a própria população. A existência de áreas altamente impermeabilizadas traz como consequência o aumento do volume escoado superficialmente, causando aumento nas vazões máximas de duas a dez vezes a vazão de pré-urbanização. Surge, então, a necessidade de alternativas que solucionem ou reduzam os problemas relacionados ao aumento do volume escoado, não somente que os transfiram para outro local. Neste sentido, pode-se aplicar as Melhores Práticas de Gestão (Best Management Practices - BMPs), medidas que servem no gerenciamento das águas pluviais em áreas urbanas, destacando-se os reservatórios de detenção. Neste contexto, o presente trabalho tem como objetivo desenvolver uma metodologia para avaliar a águas pluviais em áreas urbanas, destacando-se os reservatórios de detenção. Neste contexto, o presente trabalho tem como objetivo desenvolver uma metodologia para avaliar a
minimização do impacto hidrológico de uma bacia urbanizada para que ela se comporte de maneira hidrologicamente semelhante às condições de pré-urbanização, analisando os custos de implantação de bacias de detenção para evitar alagamentos à jusante. Para isso, fez-se uso de simulação hidrológica com modelo IPHS1 para determinação dos hidrogramas de saída dos afluentes da bacia estudada, que corresponde à bacia da Vila Santa Isabel, conhecida também como bacia Mãe D’água, de 3,39 km², localizada no município de Viamão/RS. Os resultados mostraram que a aplicação de uma técnica de otimização associada a um modelo hidrológico mostrou-se útil na avaliação dos custos de projetos de controle do escoamento pluvial urbano (bacias de detenção) para a bacia estudada. Além disso, mesmo sem a disponibilidade de dados hidrológicos observados e informaç̃os mais detalhadas acerca do processo de urbanização da área de estudo, foi possível, através da aplicação da metodologia apresentada, desenvolver cenários para análise do aumento da zona urbana e quantificação do impacto hidrológico, para que assim o problema de alagamentos fosse minimizado.

\section{Optimization for control of urban flooding: application in the Vila Isabel hidrographic Basin/Viamão/RS} \begin{abstract}
The rapid and uncontrolled urbanization, especially in developing countries, has led to significant impacts on both the environment and people themselves. The existence of highly alternatives to solve or reduce the problems related to the increased volume disposed, and not only transferring it to another location. In this context, the Best Management Practices (BMPs) can be implemented, which are measures for managing storm water in urban areas as, for example, the detention reservoirs. Thus, this work aims to develop a methodoly to evaluate the hydrological impact minimization of an urbanized basin, so that it can behave hydrologically similar to pre-urbanization conditions, analyzing deployment costs of detention basins to prevent flooding downstream. Hence, a hydrologic simulation model with IPHS1 has been used to determine the studied basin tributaries output hydrographs. The study area represents the Santa Isabel watershed, known Mãe D'água watershed, with $3,39 \mathrm{~km}^{2}$, located in Viamão/RS. The results showed that the application of an optimization technique associated with a hydrological model has proven to be useful in storm water runoff control projects costs evaluation (detention basins) for the urban study area. Moreover, even without the availability of observed hydrological data and detailed information about the process of urbanization in the study area, it was possible, through the application of the presented methodology, to develop scenarios for analyzing the urban zone expansion and quantification of hydrological impact, in order to minimize the identified problem.
\end{abstract}

Keywords: Urbanization; Detention basin; Hydrologic Simulation; Optimization; Mãe D’água Watershed.

Topic: Engenharia de Recursos Hídricos

Reviewed anonymously in the process of blind peer.
Received: 02/12/2018

Approved: 26/01/2019
Anelise Nardi Hüffner (iD)

Universidade Luterana do Brasil, Brasil

http://lattes.cnpq.br/7057825860253284

http://orcid.org/0000-0002-2560-0876

ane_huffner@yahoo.com.br

Tirzah Moreira Siqueira (iD)

Universidade Federal de Pelotas, Brasil http://lattes.cnpq.br/8413080701996952 http://orcid.org/0000-0002-6576-0217 tirzahmelo@hotmail.com

\section{Bárbara de Lima Corrêa (D)}

Universidade Federal de Pelotas, Brasil http://lattes.cnpq.br/3961220820729315 http://orcid.org/0000-0001-8029-3285

barb.lima.correa@gmail.com

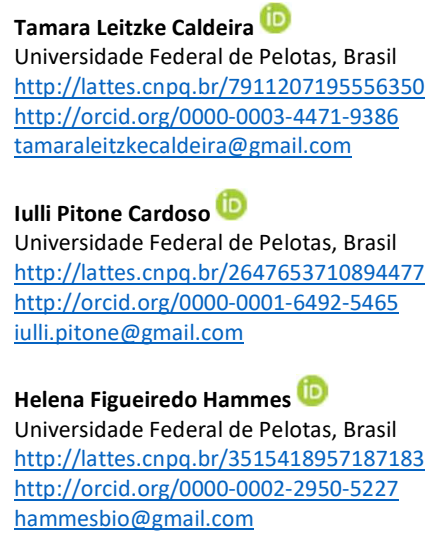

Patrícia Damasceno Ribeiro (iD Universidade Federal de Pelotas, Brasil http://lattes.cnpq.br/9031390146890126 http://orcid.org/0000-0001-8858-137X patiidamasceno@gmail.com

Referencing this:

HÜFFNER, A. N.; SIQUEIRA, T. M.; CORRÊA, B. L.; CALDEIRA, T. L.; CARDOSO, IULLI, P. C.; HAMMES, H. F.; RIBEIRO, P. D.. Otimização para controle de alagamentos urbanos: aplicação na bacia hidrográfica Vila Santa Isabel/Viamão/RS. Revista Ibero Americana de Ciências Ambientais, v.10, n.1, p.99-109, 2019. DOI: http://doi.org/10.6008/CBPC2179-6858.2019.001.0009 


\section{INTRODUÇÃO}

O crescimento rápido e descontrolado da urbanização, principalmente nos países em desenvolvimento, vem provocando impactos significativos tanto no meio ambiente quanto para a própria população. Um exemplo de impacto decorrente da urbanização é a crescente impermeabilização do solo, acarretando um aumento na frequência de alagamentos. A existência de áreas altamente impermeabilizadas traz como consequência o aumento do volume escoado superficialmente, causando acréscimo nas vazões máximas de duas a dez vezes a vazão de pré-urbanização (ROESNER et al., 2001). Surge, então, a necessidade de alternativas que solucionem ou reduzam os problemas relacionados ao aumento do volume escoado, não somente que os transfiram para outro local.

Neste sentido, podem-se aplicar as Melhores Práticas de Gestão (Best Management Practices BMPs), medidas que servem no gerenciamento das águas pluviais em áreas urbanas, destacando-se neste caso os reservatórios de detenção. Estes dispositivos podem reduzir alterações nos volumes de escoamento e são tipicamente concebidos com base em um valor de pico de vazão, onde a descarga de certa frequência não deve exceder as condições de pré-desenvolvimento (REICHOLD et al., 2010). No Brasil, como em outros países (a exemplo da França e EUA), há uma considerável experiência acumulada de empregos de bacias de detenção em contexto urbano, com início em meados do século passado. Destaca-se no país o uso deste tipo de estrutura nas regiões metropolitanas de São Paulo (SP) e Porto Alegre (RS).

Com relação ao dimensionamento e implantação de reservatórios, muitas vezes os métodos utilizados não proporcionam a obtenção de uma solução ótima com relação ao volume e/ou vazão que deve ser armazenados por estes dispositivos. A simulação tenta representar um sistema físico e prever seu comportamento sob um determinado conjunto de condições, não apresentando, praticamente, nenhuma exigência quanto à natureza do problema, a não ser a de que ele possa ser formulado matematicamente (WURBS, 1993). Já as técnicas de otimização consistem em encontrar uma solução ou um conjunto de soluções ótimas para uma determinada função ou conjunto de funções. Assim, a escolha de vários locais é necessária quando se tem bacias densamente urbanizadas e com poucos locais abertos disponíveis, podendo dessa forma trabalhar com a combinação das alternativas, para se chegar ao local ou locais mais adequados à implementação deste tipo de dispositivo.

Os modelos de otimização são formulados para encontrar os valores de um conjunto de variáveis de decisão que otimizem (maximizem e minimizem) uma função objetivo sujeita a restrições. A função objetivo e as restrições são representadas por expressões matemáticas em função das variáveis de decisão. As restrições determinam a região viável das varáveis de decisão, e as variáveis de decisão são os parâmetros cujos valores definem uma solução para o problema.

Porém, não existe um procedimento de otimização geral que possa resolver eficientemente qualquer tipo de problema. A maioria das técnicas depende da forma e propriedades matemáticas da função objetivo e restrições (VIEIRA, 2007; DAHLQUIST et al., 1974). Zehn et al. (2004) salientam que muitas pesquisas vêm sendo realizadas para resolver os problemas de manejo de águas pluviais utilizando técnicas de otimização. 
Muitos estudos mostram que isto requer que sejam formulados problemas mais simples, porém, mesmo que as soluções ótimas encontradas sejam matematicamente possíveis, seu uso prático em projetos reais é frequentemente questionável.

Diante disso, o presente trabalho tem como objetivo desenvolver uma metodologia para avaliar a minimização do impacto hidrológico de uma bacia urbanizada para que ela se comporte de maneira hidrologicamente semelhante às condições de pré-urbanização, analisando os custos de implantação de bacias de detenção para evitar alagamentos à jusante.

\section{MATERIAIS E MÉTODOS}

\section{Área de estudo}

A área de estudo é a bacia da Vila Santa Isabel, conhecida também como bacia Mãe D'água, localizada entre os municípios de Porto Alegre e Viamão, no estado do Rio Grande do Sul (Figura 1). Abriga uma das nascentes da bacia do Arroio Dilúvio, que cruza grande parte do perímetro urbano do município de Porto Alegre. Ela é composta por quatro afluentes principais e apresenta $3,39 \mathrm{~km}^{2}$ de área. 0 processo de urbanização na área da bacia remonta à década de 1950, com a formação dos primeiros loteamentos (CARDOSO, 2011). A partir dos anos de 1970, o processo de urbanização acentuou-se e a ocupação da área se deu de maneira desordenada, sem qualquer tipo de planejamento.

Assim, como a bacia está situada em uma região de cabeceira, a urbanização desordenada e consequente impermeabilização do solo, resultam em alagamentos a jusante da bacia. Isto ocorre, pois a parcela de água precipitada sobre a bacia tende a escoar mais rapidamente até o exutório, e como não há nenhum tipo de estrutura de armazenamento na área, esse escoamento acaba por contribuir para o transbordamento dos arroios a jusante.

A metodologia proposta neste trabalho consiste na elaboração de um modelo de otimização de minimização dos impactos relacionados ao escoamento superficial decorrentes da urbanização em uma bacia hidrográfica. Para tanto, foi necessária a busca de informações que possibilitassem a construção da função de minimização da diferença de pico de vazão, entre os cenários de pré-urbanização e urbanizado (atual).

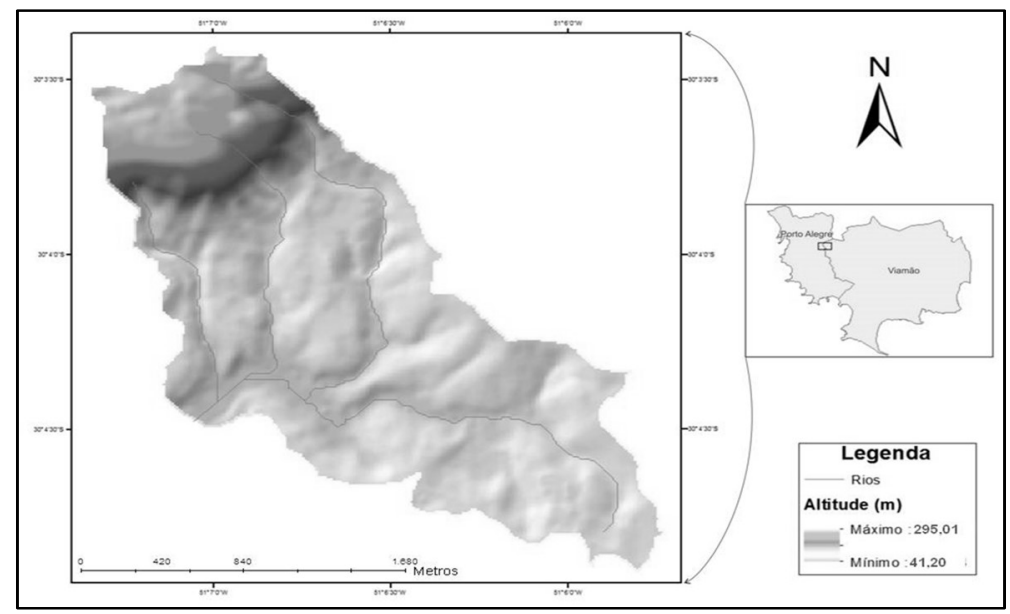

Figura 1: Localização da área de estudo - Bacia Hidrográfica da Vila Santa Isabel. 


\section{Caracterização física da bacia hidrográfica}

Para a delimitação da área de estudo foi utilizado um modelo numérico de terreno (MNT) criado a parir da base altimétrica de Porto Alegre na escala de 1:1.000, disponível no site do Laboratório de Geoprocessamento do Centro de Ecologia da UFRGS. O processo de delimitação da bacia hidrográfica e obtenção da rede de drenagem foi realizado por meio da ferramenta ArcHydro, do software ArcGis ESRI, utilizando o MNT como base para realizar o processamento. Foi feita a delimitação da bacia em 17 sub-bacias e a obtenção das características físicas da bacia e sub-bacias necessárias para este estudo, tais como: área da bacia e sub-bacias, comprimento dos afluentes, cota máxima e mínima da bacia e declividades.

\section{Determinação do impacto hidrológico}

\section{Uso do solo e obtenção do parâmetro curve number (cn)}

A caracterização da ocupação do solo foi estabelecida com base na determinação de dois cenários: pré-urbanização e urbanização atual (diagnóstico) da bacia, sendo possível a análise da evolução da urbanização na área de estudo. Estes cenários são: (a) Cenário 1 (Pré-urbanização), a imagem utilizada foi uma fotografia aérea do ano de 1972 (Metroplan) e (b) Cenário 2 (Urbanização atual), a imagem utilizada foi obtida em 2012 a partir de satélite espacial e disponibilizada no Google Earth.

Neste estudo, a estimativa do CN foi feita a partir da distribuição dos percentuais das classes de usos de solo: mata, campo, solo exposto, água e zona urbana. O tipo de solo da área de estudo é do tipo B (CAMPANA et al., 1999), contendo moderada taxa de infiltração quando molhados, sendo, principalmente, solos moderadamente profundos. Os valores de $\mathrm{CN}$ para cada tipo de classe, considerando o tipo de solo B, foram obtidos de Tucci (2009) e correspondem aos seguintes dados: classe mata, CN 60, floresta normal; classe campo, $\mathrm{CN} 60$, campo normal; classe solo exposto, $\mathrm{CN} 82$, terra; classe água, $\mathrm{CN} 98$, áreas impermeáveis; classe zona urbana, $\mathrm{CN} 85$, áreas menores que $500 \mathrm{~m}^{2}$.

\section{Cálculo do tempo de concentração}

Existem vários métodos utilizados para estimar este parâmetro hidrológico, porém, para este trabalho foram adotados os métodos do U.S. Corps of Engineer e Carter, aplicados respectivamente em bacias rurais (pré-urbanização) e urbanas (Equações 1 e 2). Essas fórmulas foram testadas com dados referentes a 29 bacias rurais e 32 bacias urbanas, sendo a Corps of Engineer ajustada para valores de área de 0,11 até $12.000 \mathrm{~km}^{2}$. Já a fórmula de Carter foi ajustada para valores de área de até $11 \mathrm{~km}^{2}$ (Silveira, 2005).

$$
\begin{gathered}
\text { Tc }=0,191 * \mathrm{~L}^{0,76} * \mathrm{~S}^{-0,19} \\
\text { Tc }=0,0977 * \mathrm{~L}^{0,6} * \mathrm{~S}^{-0,3}
\end{gathered}
$$

onde Tc é o tempo de concentração (h), L é o comprimento do rio principal $(\mathrm{km})$ e S é a declividade média $(\mathrm{m} / \mathrm{m})$.

\section{Simulação hidrológica}

Para a simulação das condições das bacias nos dois cenários descritos anteriormente foi utilizado o modelo de simulação precipitação-vazão IPHS1, desenvolvido no Instituto de Pesquisas Hidráulicas da 
Universidade Federal do Rio Grande do Sul (IPH-UFRGS). O emprego do modelo permitiu a obtenção dos hidrogramas de projeto para quantificação do impacto hidrológico na área de estudo em função do crescimento populacional e consequente impermeabilização do solo. Utilizou-se o método CN do SCS para a separação do escoamento.

Como dados de entrada do IPHS1 foram utilizados alguns parâmetros relacionados às características físicas da bacia analisada, bem como dados de precipitação e características dos solos da região. Neste caso, a precipitação foi obtida a partir da IDF do posto IPH da cidade de Porto Alegre, armazenada no banco de dados do próprio programa. As simulações foram realizadas para os tempos de retorno ( $T r$ ) de 10 e 25 anos e a duração da precipitação foi adotada como sendo duas vezes o tempo de concentração. Esses valores de Tr foram adotados segundo IPH (2005), referente ao Plano Diretor de Drenagem Urbana de Porto Alegre. Embora o indicado seja o uso de $\operatorname{Tr}$ de até 100 anos em casos onde os impactos das enchentes envolvam altos riscos à vida humana, torna-se inviável devido ao alto custo, principalmente para pequenas áreas, como é o caso do presente estudo, em que há limitação de áreas para implantação dos reservatórios. 0 impacto hidrológico foi definido como sendo a diferença entre os hidrogramas de pré-urbanização e urbanização, ou seja, a diferença de vazão de pico entre eles.

\section{Cenário de controle de cheias}

\section{Escolha das bacias de detenção e locais de implantação dos reservatórios}

Optou-se pela escolha de reservatórios de detenção aberto em grama, pois a construção de reservatórios em concreto envolve um custo maior. Os locais foram identificados a partir de imagem de satélite e visitas a campo, optando-se por áreas de contribuição maiores, para que o amortecimento das cheias tivesse mais efeito. Assim, a disponibilidade de áreas na bacia estudada restringiu-se ao leito dos arroios, visto que a bacia se encontra intensamente urbanizada e não há muitos locais livres disponíveis.

\section{Dimensionamento}

Tendo em vista que este trabalho visa à implantação de reservatórios de detenção, foi utilizado o método da curva-envelope para o dimensionamento destas estruturas, o qual é recomendado pelo Departamento de Esgotos Pluviais (DEP) de Porto Alegre para a determinação do volume de amortecimento do reservatório. O método consiste em um processo iterativo onde o volume do reservatório é dado pela máxima diferença entre os volumes afluentes e efluentes acumulados no tempo (DEP, 2005). Determinaramse os volumes de armazenamento necessários para cada reservatório e os respectivos diâmetros de descarregadores de fundo. Os volumes obtidos do método da curva-envelope foram simulados no IPHS1, utilizando o modelo de propagação em reservatórios pelo método de Puls.

\section{Combinação das alternativas}

Fez-se uma estimativa inicial das melhores alternativas de alocação dos reservatórios na bacia, realizando-se a combinação das opções de reservatório em cada afluente. Em seguida, analisaram-se as 
soluções não dominadas em termos de redução de pico de vazão e custo. A combinação das alternativas foi baseada em uma metodologia de tentativa e erro para um direcionamento na escolha das localizações dos reservatórios. A solução ótima só pode ser encontrada com um método de otimização, que foi realizado em um segundo momento.

Para a simulação hidrológica dos dispositivos de controle, considerou-se apenas um reservatório em cada afluente, isto porque a bacia hidrográfica estudada é relativamente pequena e a consideração de mais de um reservatório por afluente se tornou desnecessária e inviável. Porém, para a geração de todas as alternativas, incluiu-se, também, a opção de não haver reservatório nos afluentes, mantendo-se a vazão urbanizada de cada afluente.

Dessa forma, a partir da simulação hidrológica foram obtidos os hidrogramas de saída dos reservatórios em cada afluente e das áreas de contribuição lateral. Assim, o cálculo da diferença dos picos no exutório da bacia, resultante de cada combinação das alternativas baseou-se na seguinte equação:

$$
\mathrm{dQ}=\max \left[\left(\sum_{\mathrm{i}=1}^{\mathrm{n}} \mathrm{Qa}_{\mathrm{i}_{\mathrm{t}}}\right)+\mathrm{Q}_{\mathrm{inc}_{\mathrm{t}}}\right]-\mathrm{Q}_{\mathrm{pu}_{\mathrm{t}}}
$$

onde dQ é diferença entre os picos dos hidrogramas $\left(\mathrm{m}^{3} \cdot \mathrm{s}^{-1}\right)$ ou impacto hidrológico, $\mathrm{n}$ é o número de afluentes, Qai é o hidrograma resultante da simulação de reservatório em cada afluente $\left(\mathrm{m}^{3} \cdot \mathrm{s}^{-1}\right)$, Qinc é o hidrograma incremental $\left(\mathrm{m}^{3} \cdot \mathrm{s}^{-1}\right), \mathrm{Q}_{\text {pu }}$ é a vazão de pico de pré-urbanização $\left(\mathrm{m}^{3} \cdot \mathrm{s}^{-1}\right)$ e t é o tempo $(\mathrm{s})$.

Para estabelecer uma relação entre a diferença de pico de vazão dos hidrogramas e o custo total de implantação dos reservatórios, foi ajustada uma função linear (Equação 4):

$$
C=214,42 . V-256944
$$

onde C é o custo total de implantação ( $\mathrm{R} \$$ ) e $V$ é o volume de cada reservatório $\left(\mathrm{m}^{3}\right)$.

Estes valores de custos foram baseados no estudo feito por Carmona (2008), que analisou os custos de reservatórios públicos abertos em grama, que são estruturas executadas e mantidas com recursos do Município de Porto Alegre, sem considerar os custos de desapropriação e aquisição das áreas escolhidas para implementação dos reservatórios. Utilizando a função ajustada (Equação 4), estimou-se o custo total de cada alternativa de alocação de reservatórios na bacia em função do volume de armazenamento. Os locais que apresentaram as melhores relações custo-redução de pico foram considerados no modelo de otimização.

\section{Modelo de otimização}

O modelo de otimização foi desenvolvido em linguagem computacional otimizada do Matlab utilizando-se da função fmincon. Esta por sua vez, resolve problemas de maximização e minimização de funções-objetivo podendo considerar restrições do tipo lineares e não lineares. A função objetivo foi definida de modo a minimizar a diferença entre o pico de vazão dos hidrogramas decorrentes da simulação dos cenários, ou seja:

$$
\operatorname{MIN~dQ}=\left[\sum_{\mathrm{i}=1}^{\mathrm{n}} \mathrm{Qp}_{\mathrm{i}}+\mathrm{Q}_{\mathrm{p}_{\mathrm{inc}}}\right]-\mathrm{Q}_{\mathrm{pu}}
$$

onde dQ é a diferença de pico entre os hidrogramas $\left(\mathrm{m}^{3} \cdot \mathrm{s}^{-1}\right), \mathrm{n}$ é o número de afluentes, $\mathrm{Q}_{\mathrm{p}}$ é a vazão de pico em cada

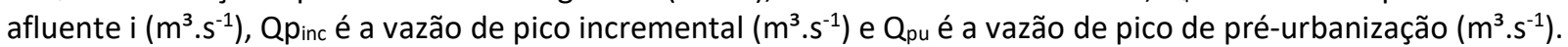

Conforme visto anteriormente, para cada localização selecionada os reservatórios foram dimensionados, obtendo-se assim os seus volumes de armazenamento, e simulados hidrologicamente, 
gerando os hidrogramas de saída em cada afluente. Dos hidrogramas, extraiu-se a vazão de pico correspondente ao volume simulado. Ajustou-se, então, para cada reservatório, uma equação do tipo:

$$
Q p_{i}=f(V)
$$

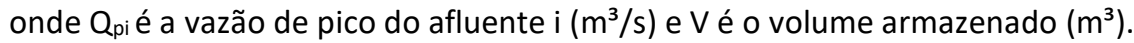

Este ajuste foi necessário, pois a função objetivo depende de $Q_{p}$ e as restrições são em função de custo ou volume. Salienta-se aqui a simplificação de se considerar que as vazões de pico ocorrem ao mesmo tempo, devido ao fato de não ter sido possível o ajuste de funções com o hidrograma resultante de cada volume simulado. Foram consideradas as seguintes restrições sobre a função-objetivo: a) Restrição de volume: A restrição de volume indica que cada reservatório pode variar entre um mínimo e máximo, ou seja:

$$
\mathrm{V}_{\min _{\mathrm{i}}} \leq \mathrm{V}_{\mathrm{i}} \leq \mathrm{V}_{\text {máxi }_{\mathrm{i}}}
$$

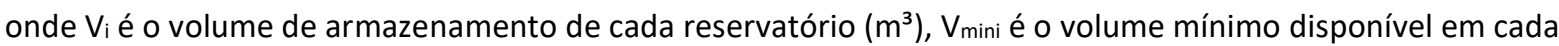
reservatório $\left(\mathrm{m}^{3}\right)$ e $V_{\text {máxi }}$ é o volume máximo a ser armazenado em cada reservatório $\left(\mathrm{m}^{3}\right)$. Os limites superiores foram baseados nos resultados obtidos do dimensionamento dos reservatórios (Método da curva envelope).

b) Restrição de custo: A restrição de custo está associada à quantidade de dinheiro disponível para a implantação dos reservatórios. Logo, $V_{\text {máx }} \leq C_{\text {máx }} / C_{0}$, ou seja:

$$
\mathrm{V}_{1}+\mathrm{V}_{2}+\cdots+\mathrm{V}_{\mathrm{n}} \leq \frac{\mathrm{C}_{\text {máx }}}{\mathrm{C}_{\mathrm{o}}}
$$

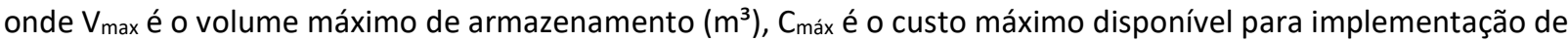
reservatórios (R\$), Co é o custo unitário $\left(R \$ / \mathrm{m}^{3}\right)$, definido a partir da estimativa de custo em função do volume de reservatório, e n é o número de reservatórios.

A restrição de custo orçamentário para implantação dos reservatórios variou de $\mathrm{R} \$ 1.000 .000,00$ até $\mathrm{R} \$ 9.000 .000,00$ (com base na etapa combinação das alternativas, descrita anteriormente), sendo que o volume de armazenamento máximo para o conjunto de reservatórios foi associado à razão de custo máximo ( $R \$)$ e custo unitário ( $\left.R \$ 163,00 \mathrm{~m}^{-3}\right)$.

Diferentes valores de custo máximo foram utilizados objetivando avaliar a quantidade e o volume dos reservatórios necessários para se atingir uma redução de pico de vazão onde a bacia urbanizada tenha um comportamento hidrológico semelhante às condições de pré-urbanização. Além de analisar os investimentos mínimo e máximo em torno desta redução, definindo uma margem de tolerância para a redução da vazão de pico. Restrição de imagem das funções ajustadas: Esta restrição assegura que as vazões obtidas a partir do ajuste sejam sempre positivas, já que valores negativos não possuem sentido físico neste estudo:

$$
\mathrm{Q}_{\mathrm{pi}}=\mathrm{f}\left(\mathrm{V}_{\mathrm{i}}\right) \geq 0
$$

onde $Q_{p}$ é a vazão de pico $\left(\mathrm{m}^{3} / \mathrm{s}\right)$ e $V_{i}$ é o volume de armazenamento de cada reservatório $\left(\mathrm{m}^{3}\right)$.

\section{RESULTADOS}

\section{Determinação do impacto hidrológico}

Devido à dificuldade de encontrar informações e imagens mais antigas da área de estudo, fez-se então a classificação da imagem do ano de 1972 e, para reproduzir um cenário de pré-urbanização, excluíram-se os valores da zona urbana, fazendo com que fossem divididos entre as demais classes (Tabela 1). Na Tabela 2 são apresentados os tempos de concentração de cada sub-bacia, que foram utilizados na 
simulação hidrológica, obtidos a partir das Equações 1 e 2, respectivamente. $O$ parâmetro $C N$ foi estimado de acordo com os valores de usos do solo obtidos onde, na Tabela 2, são demonstrados os valores estimados para cada sub-bacia nos cenários de pré-urbanização (excluindo-se a área urbana) e urbanização, respectivamente.

Os valores de tempo de concentração, juntamente com os resultados das etapas anteriores, foram utilizados no modelo hidrológico para a simulação dos cenários para quantificação do impacto hidrológico para os tempos de retorno ( $\operatorname{Tr}$ ) de 10 e 25 anos. Para um $\operatorname{Tr}$ de 10 anos, obteve-se uma vazão de pico de préurbanização de $2,23 \mathrm{~m}^{3} \mathrm{~s}^{-1}$, enquanto que para o cenário de urbanização, o pico foi de $7,53 \mathrm{~m}^{3} \mathrm{~s}^{-1}$. A vazão de urbanização representa aproximadamente 3,5 vezes a vazão de pré-urbanização, indicando que há impacto hidrológico de $5,30 \mathrm{~m}^{3} \cdot \mathrm{s}^{-1}$. No caso de uma chuva de $\operatorname{Tr}$ de 25 anos, a vazão de pico para o cenário de urbanização foi $13,11 \mathrm{~m}^{3} \cdot \mathrm{s}^{-1}$. Isto representa aproximadamente 6 vezes a vazão de pré-urbanização, com um impacto hidrológico de $10,88 \mathrm{~m}^{3} \cdot \mathrm{s}^{-1}$.

Tabela 1: Tipos de Uso do Solo (\%).

\begin{tabular}{|l|l|l|l|l|l|l|l|l|l|l|}
\hline \multirow{2}{*}{ Sub-bacia } & \multicolumn{4}{|l}{ Tipos de Uso do Solo “Pré-Urbanização" (\%) } & \multicolumn{4}{l|}{ Tipos de Uso do Solo “Urbanização" (\%) } \\
\cline { 2 - 10 } & Mata & Campo & Solo Exposto & Água & Mata & Campo & Solo Exposto & Água & Zona Urbana \\
\hline 1 & 0 & 100 & 0 & 0 & 62,41 & 34,08 & 0 & 2,06 & 1,46 \\
\hline 2 & 6,94 & 37,62 & 5,44 & 0 & 43,14 & 19,49 & 5,82 & 3,40 & 28,15 \\
\hline 3 & 0,12 & 72,52 & 4,50 & 2,85 & 23,53 & 17,92 & 2,00 & 7,12 & 49,43 \\
\hline 4 & 2,29 & 57,71 & 0 & 0 & 64,52 & 30,48 & 0 & 4,83 & 0,17 \\
\hline 5 & 3,32 & 15,36 & 11,33 & 0 & 27,52 & 24,04 & 7,18 & 2,55 & 38,72 \\
\hline 6 & 0,35 & 65,66 & 13,99 & 0 & 3,28 & 23,03 & 17,82 & 0,69 & 55,18 \\
\hline 7 & 6,97 & 61,18 & 17,32 & 4,53 & 5,67 & 25,01 & 7,98 & 1,98 & 59,36 \\
\hline 8 & 2,22 & 47,78 & 0 & 0 & 17,44 & 76,32 & 0,00 & 0,56 & 5,68 \\
\hline 9 & 9,46 & 29,39 & 21,14 & 0 & 19,87 & 29,69 & 8,82 & 1,47 & 40,15 \\
\hline 10 & 2,55 & 26,83 & 50,62 & 0 & 3,77 & 22,56 & 11,26 & 0,79 & 61,62 \\
\hline 11 & 8,37 & 37,31 & 21,60 & 2,72 & 13,60 & 16,20 & 9,03 & 7,28 & 53,89 \\
\hline 12 & 7,89 & 52,63 & 29,47 & 0 & 2,64 & 24,50 & 14,66 & 0,21 & 57,99 \\
\hline 13 & 21,19 & 43,10 & 2,28 & 33,43 & 58,57 & 9,00 & 3,16 & 15,58 & 13,70 \\
\hline 14 & 4,63 & 33,16 & 12,21 & 0 & 25,89 & 20,95 & 6,35 & 3,59 & 43,22 \\
\hline 15 & $7, .23$ & 55,14 & 17,63 & 0 & 17,13 & 23,69 & 9,65 & 3,16 & 46,38 \\
\hline 16 & 6,26 & 38,93 & 24,81 & 0 & 10,76 & 27,84 & 11,88 & 2,12 & 47,39 \\
\hline 17 & 0 & 87,61 & 12,39 & 0 & 5,24 & 33,92 & 9,79 & 3,35 & 47,69 \\
\hline
\end{tabular}

Tabela 2: Análise da ocupação urbana (\%) e valores de CN.

\begin{tabular}{|l|l|l|l|l|l|l|l|}
\hline Sub-bacia & Pré-urbanização & \multirow{2}{*}{2012} & & \multicolumn{2}{l|}{ CN } & \multicolumn{2}{l|}{ Tempo de concentração } \\
\cline { 3 - 7 } & & & Pré-urbanização & Urbanização & \multicolumn{2}{l|}{ Pré-urbanização } & Urbanização \\
\hline 1 & 0 & 1,46 & 60 & 61,2 & 4,47 & 4,02 \\
\hline 2 & 10,56 & 28,15 & 61,2 & 69,6 & 7,58 & 5,00 \\
\hline 3 & 0 & 49,43 & 62 & 75,5 & 5,31 & 4,50 \\
\hline 4 & 0 & 0,17 & 60 & 61,9 & 5,65 & 4,40 \\
\hline 5 & 10,00 & 38,72 & 65,5 & 72,2 & 5,18 & 3,70 \\
\hline 6 & 17,31 & 55,18 & 63 & 78 & 5,85 & 4,65 \\
\hline 7 & 19,76 & 59,36 & 65,5 & 77,4 & 6,58 & 5,17 \\
\hline 8 & 0 & 5,68 & 60 & 61,6 & 3,37 & 2,67 \\
\hline 9 & 22,84 & 40,15 & 64 & 72,5 & 5,52 & 3,89 \\
\hline 10 & 53,09 & 61,62 & 71,1 & 78,2 & 12,35 & 9,41 \\
\hline 11 & 36,16 & 53,89 & 65,8 & 78,2 & 11,73 & 9,06 \\
\hline 12 & 29,1 & 57,99 & 66,5 & 77,8 & 7,18 & 6,00 \\
\hline 13 & 0 & 13,7 & 73,2 & 70 & 10,19 & 10,63 \\
\hline 14 & 23,32 & 43,22 & 62,7 & 73,6 & 9,15 & 7,26 \\
\hline 15 & 36,77 & 46,38 & 63,9 & 75 & 16,29 & 12,32 \\
\hline 16 & 49,93 & 47,39 & 65,5 & 75,3 & 9,51 & \\
\hline 17 & 54,8 & 47,69 & 62,7 & 75,4 & 8,13 & 7,32 \\
\hline
\end{tabular}




\section{Escolha e combinação das alternativas dos locais para implantação das bacias de detenção}

Destaca-se que, como em projetos é inviável dimensionar uma obra para diferentes períodos de

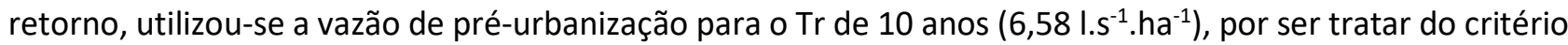
utilizado para o dimensionamento da maioria das obras de drenagem (TASSI, 2005). Para o cenário de Tr de 10 anos, assumiram-se como melhores resultados aqueles que obtiveram uma diferença de pico máxima de $0,98 \mathrm{~m}^{3} \cdot \mathrm{s}^{-1}$, ou seja, que obtiveram uma redução de pelo menos $57 \%$ da vazão de urbanização. Isto porque esses valores se referem sempre a mesma combinação de reservatórios. Esses resultados tiveram um custo mínimo de $\mathrm{R} \$ 2.200 .000,00$. Da mesma forma, para o $\operatorname{Tr}$ de 25 anos, considerou-se uma diferença de pico máxima de $3,1 \mathrm{~m}^{3} \cdot \mathrm{s}^{-1}$, ou seja, que obtiveram uma redução de pelo menos $59 \%$ da vazão de urbanização, resultando em um custo mínimo de R\$ 2.500.000,00.

Observa-se que a redução de pico não chega a $0 \mathrm{~m}^{3} \cdot \mathrm{s}^{-1}$, isto é, os volumes dimensionados para um $\mathrm{Tr}$ de 25 anos não foram suficientes para armazenar uma chuva dessa magnitude. Isto pode ser explicado devido ao fato de que, para o dimensionamento dos reservatórios para este tempo de retorno, manteve-se a vazão máxima específica $\left(6.58 \mathrm{l} . \mathrm{s}^{-1} \cdot \mathrm{ha}^{-1}\right)$ para o tempo de retorno de 10 anos.

Para os dois $\operatorname{Tr}$ analisados, todas as soluções não dominadas que obtiveram maiores reduções de vazão de pico, ou seja, que apresentaram menor dQ utilizaram os reservatórios 1 no afluente 1, 3 no afluente 2, 5 no afluente 3 e 6 no afluente 4. Os reservatórios 2, 4, 7 não foram utilizados nestas alternativas. Para o $\operatorname{Tr}$ de 10 anos, apenas os reservatórios 1, 3, 5 e 6 foram utilizados no modelo de otimização por apresentarem uma dQ máxima de $0,98 \mathrm{~m}^{3} \cdot \mathrm{s}^{-1}$. Para o $\operatorname{Tr}$ de 25 anos, a dQ máxima foi de $3,1 \mathrm{~m}^{3} \cdot \mathrm{s}^{-1}$, considerando-se os mesmos reservatórios para o tempo de retorno de 10 anos.

\section{Otimização dos volumes dos reservatórios}

A partir da identificação da melhor combinação, ajustou-se uma função do tipo polinomial de $3^{\circ}$ grau, apresentada pela Equação 10, para os reservatórios de amortecimento que apresentaram os melhores resultados (1, 3, 5 e 6$)$ em cada afluente para ambos os tempos de retorno (10 e 25 anos).

$$
\mathrm{Q}_{\mathrm{p}}(\mathrm{V})=a \mathrm{~V}^{3}+\mathrm{bV}^{2}+c \mathrm{~V}+\mathrm{d}
$$

onde $Q_{p}$ é a vazão de pico $\left(\mathrm{m}^{3} \cdot \mathrm{s}^{-1}\right)$, $V$ é o volume armazenado $\left(\mathrm{m}^{3}\right)$, a, b, c e d são coeficientes de ajuste $\left(\mathrm{m}^{-6} \cdot \mathrm{s}^{-1}, \mathrm{~m}^{-3} \cdot \mathrm{s}^{-1}\right.$

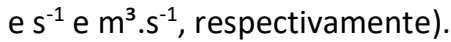

Os limites inferiores e superiores dos volumes individuais de cada reservatório de detenção são mostrados na Tabela 3, conforme as restrições de custo apresentadas anteriormente.

Tabela 3: Restrições de volumes de armazenamento.

\begin{tabular}{|l|l|l|}
\hline Reservatórios & T 10 anos & T 25 anos \\
\hline R1 & $0 \mathrm{~m}^{3} \leq \mathrm{V}_{1} \leq 6.411,68 \mathrm{~m}^{3}$ & $0 \mathrm{~m}^{3} \leq \mathrm{V}_{1} \leq 10.000 \mathrm{~m}^{3}$ \\
\hline R3 & $0 \mathrm{~m}^{3} \leq \mathrm{V}_{2} \leq 7.995,52 \mathrm{~m}^{3}$ & $0 \mathrm{~m}^{3} \leq \mathrm{V}_{2} \leq 12.000 \mathrm{~m}^{3}$ \\
\hline R5 & $0 \mathrm{~m}^{3} \leq \mathrm{V}_{3} \leq 11.939,07 \mathrm{~m}^{3}$ & $0 \mathrm{~m}^{3} \leq \mathrm{V}_{3} \leq 17.000 \mathrm{~m}^{3}$ \\
\hline R6 & $0 \mathrm{~m}^{3} \leq \mathrm{V}_{4} \leq 13.438,78 \mathrm{~m}^{3}$ & $0 \mathrm{~m}^{3} \leq \mathrm{V}_{4} \leq 19.000 \mathrm{~m}^{3}$ \\
\hline
\end{tabular}

O resultado desejado corresponde a dQ igual a 0 , pois este valor significa que a vazão de pico no exutório da bacia corresponde à vazão de pico de pré-urbanização. Porém, para uma análise mais completa 
em termos de recurso financeiro investido, trabalhou-se dentro de uma margem de tolerância para a redução da vazão de pico, ou seja, analisaram-se investimentos mínimo e máximo em torno da dQ=0 (Tabela 4).

Tabela 4: Análise dos investimentos para redução de vazão.

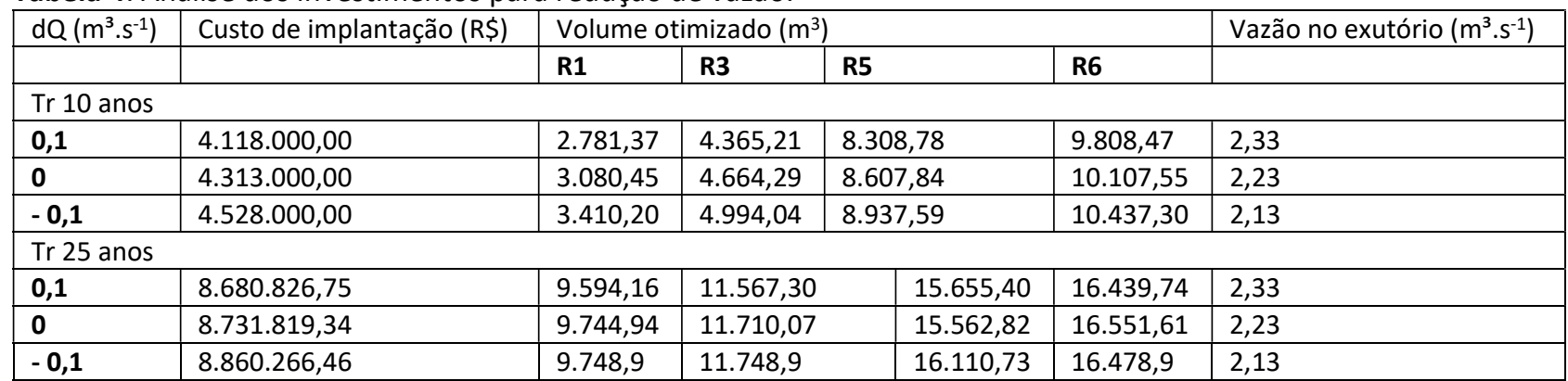

\section{DISCUSSÃO}

Assim, analisando-se as Tabelas 1 e 2, observa-se que no ano de 1972 já se evidenciava um processo de expansão da zona urbana na área de estudo, ou seja, 21\% da bacia era urbanizada. No ano de 2012 (cenário de urbanização), a área urbanizada é maior, representando $38,25 \%$ de toda a área estudada. Observa-se que na sub-bacia 13, com valores de CN 73,2 no cenário de pré-urbanização e de 70 para o cenário de urbanização, há diminuição do valor do $\mathrm{CN}$, isto porque constatou-se que nesta sub-bacia houve o crescimento de vegetação devido à existência de uma barragem no local, o que fez com que na classificação da imagem de 2012 o percentual de mata fosse maior que para o cenário anterior.

Assim sendo, é possível afirmar que a imposição de restrições de vários cenários de custos máximos resultou em soluções satisfatórias, atingindo o objetivo proposto neste trabalho. Isto é, pode-se se fazer uma análise acerca de qual é o melhor investimento a ser feito na área de estudo, de acordo com o recurso financeiro disponível, e saber qual é a dQ resultante.

Desta forma, os resultados apresentados na Tabela 4 representa uma faixa de valores (aos quais se associam os volumes de quatro reservatórios) que garantem que não ocorrerão alagamentos a jusante da área de estudo para um tempo de retorno de 10 e 25 anos, como consequência do amortecimento gerado por esses reservatórios.

Porém, cabe ressaltar que para a implantação desses reservatórios de amortecimento, é importante que sejam calculados os diâmetros comerciais dos descarregadores de fundo e seus respectivos volumes, para que assim se tenha valores mais próximos da realidade, uma vez que este estudo só faz uma análise acerca dos resultados obtidos na otimização.

\section{CONCLUSÕES}

A prática corrente adotada na determinação de volumes da reservação em planos de drenagem urbana é de avaliar individualmente cada sub-bacia, não havendo uma análise de combinações distintas da alocação dos volumes que confiram um efeito sinérgico semelhante. Porém, com a otimização dos custos de implantação, essa avaliação foi possível. A aplicação de uma técnica de otimização associada a um modelo hidrológico mostrou-se útil na avaliação dos custos de projetos de controle do escoamento pluvial urbano 
(bacias de detenção) para a bacia estudada, podendo servir como material de consulta para o Poder Público e Privado.

O estudo buscou validar a hipótese de aplicabilidade da metodologia a bacias urbanas através do estudo de caso na região metropolitana de Porto Alegre, especificamente no município de Viamão. Os resultados obtidos mostraram que, mesmo sem disponibilidade de dados hidrológicos observados e informações mais detalhadas acerca do processo de urbanização da área de estudo, é possível, através da aplicação da metodologia apresentada, desenvolver cenários para análise do aumento da zona urbana e quantificação do impacto hidrológico, para que assim o problema identificado seja minimizado.

Entre os aspectos que devem ser considerados em trabalhos futuros, espera-se que sejam levantados dados acerca do valor de terrenos em bacias urbanizadas, para que dessa forma seja feita uma análise mais ampla acerca dos custos que envolvem os reservatórios de detenção. Além disso, sugere-se a aplicação desta metodologia utilizando-se outras medidas de controle de escoamento, além dos reservatórios de detenção. Pode-se, também, considerar a otimização de um conjunto de melhores práticas de gestão (Best Management Practices - BMPs), avaliando os custos de implantação dessas medidas, e compará-los com os valores encontrados somente para bacias de detenção.

\section{REFERÊNCIAS}

CAMPANA, N. A.; TUCCI, C. E. M.. Previsão da vazão em macrobacias urbanas: arroio dilúvio em Porto Alegre. Revista Brasileira de Recursos Hídricos, Porto Alegre, v.4, n.1, p.19-33, 1999. DOI: http://doi.org/10.21168/rbrh.v4n1.p19-33

CARDOSO, A. R.. Evolução urbana e o enriquecimento de sedimentos por metais-traço na Barragem Mãe D’água, sub-bacia do Arroio Dilúvio, RMPA/RS. Dissertação (Mestrado em Recursos Hídricos e Saneamento Ambiental) Universidade Federal do Rio Grande do Sul, Porto Alegre, 2011.

CARMONA, M. V. C.. Gestão da drenagem Urbana em Porto Alegre/RS. Monografia (Especialização em Gestão Integrada em Saneamento) Universidade de Brasília, Brasília, 2008.

DAHLQUIST, G.; BJORCK, A.. Numerical Methods. New Jersey: Prentice-Hall, 1974.

RIO GRANDE DO SUL. Caderno de Encargos do Departamento de Esgotos Pluviais. Porto Alegre: DEP, 2005.

ROESNER, L. A.; BLEDSOE, B. P.; BRASHEAR, R. W.. Are bestmanagement-practices criteria really environmentally friendly?. Journal of Water Resources Planning and Management, v.127, p.150-154, 2001.

REICHOLD, L.; ZECHMAN, E. M.; BRILL, E. D.; HOLMES, H.. Simulation-Optimization Framework to Support Sustainable
Watershed Development by Mimicking the Predevelopment Flow Regime. Journal of Water Resources Planning and Management, v.136, n.3, p.150-154, 2010.

SILVEIRA, A. L. L.. Desempenho de fórmulas de tempo de concentração em bacias urbanas e rurais. Revista Brasileira de Recursos Hídricos, v.10, n.1, p.5-23, 2005. DOI: http://doi.org/10.21168/rbrh.v10n1.p5-29

TASSI, R.. Comparação de duas metodologias para a determinação do volume de detenção em pequenas bacias urbanas: o caso de Porto Alegre/RS. In: CONGRESO NACIONAL DEL AGUA, 20. Anais. Mendoza: INA, 2005.

TUCCI, C. E. M.. Hidrologia ciência e aplicação. 4 ed. Porto Alegre: Editora da UFRGS/ABRH, 2009.

VIEIRA, A. S.. Um modelo de simulação, via programação linear sequencial, para sistema de recursos hídricos. Dissertação (Mestrado em Engenharia Civil e Ambiental) Universidade Federal de Campina Grande, Campina Grande, 2007.

ZEHN, X-Y. J.; YU, S. L.; LIN, J-Y.. Optimal location and sizing of stormwater basins at watershed scale. Journal of Water Resources Planning and Management, v.130, n.4, 2004.

WURBS, R. A.. Reservoir-system simulation and optimization models. Journal of Water Resources Planning and Management, v.119, n.4, p.455-472, 1993.

A CBPC - Companhia Brasileira de Produção Científica (CNPJ: 11.221.422/0001-03) detém os direitos materiais desta publicação. Os direitos referem-se à publicação do trabalho em qualquer parte do mundo, incluindo os direitos às renovaç̃̃es, expansões e disseminações da contribuição, bem como outros direitos subsidiários. Todos os trabalhos publicados eletronicamente poderão posteriormente ser publicados em coletâneas impressas sob coordenação da Sustenere Publishing, da Companhia Brasileira de Produção Científica e seus parceiros autorizados. Os (as) autores (as) preservam os direitos autorais, mas não têm permissão para a publicação da contribuição em outro meio, impresso ou digital, em português ou em tradução. 